\title{
FORUM
}

\author{
Rita Schäfer
}

\section{Systematische Gender-Perspektiven - Schlüssel für Frieden und Sicherheit?}

\section{Frauen als Friedensstifterinnen}

Die Verleihung des Friedensnobelpreises 2011 an Ellen Johnson-Sirleaf und Leymah Gbowee aus Liberia sowie Tawakkul Karman aus dem Jemen setzte Zeichen. Sie betonte die politische Bedeutung von Frauen als Akteurinnen in Friedensprozessen und nahm auf die Resolution 1325 des UN-Sicherheitsrats zu »Frauen, Frieden und Sicherheit« Bezug. Diese Ende Oktober 2000 einstimmig verabschiedete Resolution sowie ihre Folgeresolutionen 1820, 1888, 1889 und 1960 aus den Jahren 2008 bis 2010 forderten politische Entscheidungsträger auf internationaler und nationaler Ebene zum Handeln auf. Die Fokussierung dieser Resolutionen auf Frauen als Friedensstifterinnen und die Auseinandersetzung der UN mit sexualisierter Gewalt als friedens- und sicherheitspolitisches Problem beförderte Diskussionen in Organisationen und Institutionen. Dabei ging es einerseits darum, die Ausrichtung auf Frauen im Kontext komplexer Gender-Ansätze zu erörtern, und andererseits stellte sich die Herausforderung, sexuelles Fehlverhalten durch das eigene Personal zu verhindern.

Auch Wissenschaftlerinnen, die zu Frauen- oder Gender-Themen in der Friedensund Konfliktforschung arbeiten, nutzten den zehnten Jahrestag der Verabschiedung der UN-Resolution 1325 im Oktober 2010, um sich genauer mit dem politisch brisanten Thema auseinanderzusetzen. So entstanden 2010 und 2011 etliche themenrelevante Neuerscheinungen, von denen hier vier Sammelbände vorgestellt werden, die unterschiedliche konzeptionelle Zugänge innerhalb der breit gefächerten Debatte bieten. ${ }^{1}$ Sie illustrieren exemplarisch die fortschreitende Divergenz zwischen einer Frauenforschung, die weitgehend die der Resolution 1325 zugrunde liegende Ausrichtung auf geschlechtsspezifische Rollenzuschreibungen übernimmt, und in-

1 Ausführliche Literaturlisten, die den Forschungsstand dokumentieren, bieten die Webseite www.frauen-und-kriege-afrika.de und www.gwi-boell.de. 
novativen Gender-Ansätzen, die polarisierende Geschlechterstereotypen im Kontext der internationalen Friedenssicherung hinterfragen.

Engels, Bettina / Gayer, Corinna (Hrsg.): Geschlechterverhältnisse, Frieden und Konflikt, Feministische Denkanstöße für die Friedens- und Konfliktforschung. Baden-Baden: Nomos Verlagsgesellschaft 2011.

Kuehnast, Kathleen / de Jonge Oudraat, Chantal / Hernes, Helga (eds.): Women and War-Power and Protection in the 21st Century. Washington D.C.: USIP Press 2010.

Olonisakin, Funmi / Okech, Awino (ed.): Women and Security Governance in Africa. Cape Town: Pambazuka Press 2011.

Olonisakin, Funmi / Barnes, Karen / Ikpe, Eka (eds.): Women, Peace and Security, Translating Policy into Practice. London: Routledge 2010.

\section{Resolution 1325 - erste Bilanzen aus Sicht der Frauen- und Gender- Forschung}

Die Autorinnen und Autoren des Sammelbands »Women and War« (2010), herausgegeben von Kathleen Kuehnast, Chantal de Jonge Oudraat und Helga Hernes, nehmen ausdrücklich auf die UN-Resolution 1325 Bezug. Sie schätzen die Nachkriegszeit als Phase ein, in der Veränderungen der Geschlechterordnungen möglich sind. Deshalb kritisieren sie, dass die dazu notwendigen Schritte trotz der UN-Resolution 1325 und ihrer Folgeresolutionen, die einen Referenzrahmen böten, oft nicht unternommen werden. Die insgesamt sechs Beiträge sind Ergebnis eines Diskussionsprozesses von Mitarbeiterinnen des United States Institute of Peace und des Peace Research Institute of Oslo. Die friedenspolitisch engagierte Forscherin Sanam Anderlini zeichnet in ihrem Beitrag »Translating Global Agreements into National and Local Committments « (2010) die politische Lobbyarbeit von Frauenrechtsaktivistinnen in den 1990er Jahren für die Verabschiedung der UN-Resolution 1325 nach. Angesichts der mangelnden Umsetzung dieser Resolution verlangt sie Frauenquoten, verbesserte Gender-Trainings sowie härtere Strafen bei sexuellem Fehlverhalten von Blauhelmsoldaten. Zudem schlägt Anderlini vor, Ex-Kämpferinnen aus Guerillaorganisationen speziell für UN-Friedensmissionen auszubilden. Währenddessen illustriert Elisabeth Wood in ihren Aufsatz »Rape is not Inevitable during War« (2010) die Verbreitung von Vergewaltigungen in unterschiedlichen Kriegsformen. Inger Skjelsbaek widmet sich in ihrem Beitrag zu »Sexual Violence in the Post-Yugoslav War« (2010) der strafrechtlichen Aufarbeitung dieser Form der Kriegsgewalt. Unter dem Titel »Impact of Violent Conflict on Women's Eco- 
nomic Opportunities« dokumentieren Tilman Brück und Marc Vothknecht (2010) die negativen Auswirkungen gewaltsamer Konflikte auf die wirtschaftlichen Kapazitäten von Frauen.

Allen gemeinsam sind die empirische Ausrichtung ihrer Erläuterungen sowie die Situationsanalyse der Problemlage von Frauen während und nach Kriegen. Sie verlangen, dass die Machtungleichheit zwischen den Geschlechtern als politisches Problem erkannt wird, das die Neustrukturierung von Nachkriegsgesellschaften und nachhaltige Friedensprozesse beeinträchtigt. »Women and War« fokussiert stärker als andere themenrelevante Publikationen auf Frauen und referiert zudem im Wesentlichen US-amerikanische und europäische Diskurse.

Ähnlich wie die am Sammelband »Women and War« beteiligten WissenschaftlerInnen konzentrieren sich auch die Autorinnen und Autoren in »Women, Peace and Security«, das im gleichen Jahr von Funmui Olonisakin, Karen Barnes und Eka Ikpe (2010) herausgegeben wurde, vorrangig auf die UN-Resolution 1325 und die darin enthaltenen Transformationspotenziale nach Kriegen. Sie kritisieren die konzeptionellen Fallstricke dieser Resolution und deren Folgen für die Praxis, etwa die Betrachtung von Frauen als homogene Gruppe und die Vernachlässigung kriegsgeprägter Männlichkeit. Aus ihrer Sicht ist die Verbindung von wissenschaftlichen Studien mit politischen Stellungnahmen, die Missstände anprangern, kein Nachteil für die Wissenschaft; im Gegenteil, gerade nach Kriegen hätten Forschende die Aufgabe, neue Machthaber sowie politische und gesellschaftliche Prozesse kritisch zu beobachten und Fehlentwicklungen offen zu legen.

Der Sammelband greift die zentralen Begriffe der UN-Resolution 1325 und ihrer Folgresolutionen auf; die Beiträge analysieren mehrheitlich die Umsetzung der normativen und sicherheitspolitischen Vorgaben in der Praxis. Dazu stellen die Autorinnen nationale Aktionspläne zur Umsetzung der Resolution in Nachkriegsländern vor. Zudem ordnen sie Gender-Differenzen, die Distinktionen und Interessendivergenzen zwischen Frauen und zwischen Männern unterschiedlichen Status, Machtund Ressourcenzugangs einschließen, während bzw. nach Kriegen in übergreifende politische und sozio-ökonomische Machtfragen ein.

Die meisten Autorinnen von »Women, Peace and Security « arbeiten in Friedensund Entwicklungsorganisationen in den Ländern, über die sie berichten, wie Sumie Nakaya mit ihrer Analyse »Women and Gender Issues in Peacebuilding. Lessons Learned from Timor-Leste « (2010) und Lesley Abdela mit ihrem Beitrag »Nepal and the Implementation of UNSCR 1325« (2010). Sie belegen, dass die Umsetzung der UN-Resolution 1325 viele Leerstellen aufweist. Die Probleme beginnen mit den strukturellen Mängeln von UN-Friedensmissionen, konkret dem Fehlverhalten von Blauhelmsoldaten und der mangelnden Berücksichtigung situationsspezifischer 
Gewaltformen; sie umfassen den begrenzten politischen Willen der Nachkriegsregierungen und enden mit der unzureichenden Auseinandersetzung mit lokalen Friedensinitiativen. So werden Chancen für Strukturreformen verspielt und geschlechtsspezifische Gewaltmuster ungebrochen in den Nachkriegsalltag übernommen.

\section{Systematische Gender-Perspektiven}

Funmi Olonisakin, eine der Herausgeberinnen des Sammelbands »Women, Peace and Security«, zeigt in der ebenfalls von ihr mitherausgegebenen Publikation »Women and Security Governance in Africa" (Olonisakin/Okech 2011), wie Friedensprozesse durch die Fortsetzung martialischer Maskulinitätszuschreibungen gefährdet werden und inwieweit es Auswege aus Gewaltmustern für Ex-Kämpfer auf lokaler und nationaler Ebene gibt. Funmi Olonisakin, die an afrikanischen und europäischen Friedensinstitutionen arbeitet, hat ein Netzwerk afrikanischer Friedensforscherinnen aufgebaut; gemeinsam mit dort engagierten Wissenschaftlerinnen nutzt sie Gender als politisch relevanten Analysebegriff. Dieser beinhaltet die systematische Beachtung von martialisch aufgeladener Männlichkeit, einem Strukturproblem, das die meisten Friedens- und Sicherheitsplaner übersehen. Auch für die Konfliktforschung eröffnet er neue Perspektiven. Die differenzierte und lokalspezifische Analyse der Übernahme kriegerischer Gewaltmuster als Inbegriff männlicher Selbstbilder und maskulinen Machthandelns in den Nachkriegsalltag ermöglicht es, die Gewaltlogiken in labilen Post-Konfliktgesellschaften in einer großen Tiefenschärfe auszuleuchten.

Im gesamten Sammelband »Women and Security Governance in Africa«, der auch Fallstudien zu Sierra Leone (Sidi Bah 2011) und Mosambik (Scanlon/Nhalevilo 2011), zur ECOWAS (Ikpe 2011) und zu Afrikanischen Union (Murithi 2011) enthält, beschränken sich die AutorenInnen nicht darauf, die geschlechtsspezifischen Gewaltmuster von Ex-Kämpfern und demobilisierten Soldaten zu untersuchen. Vielmehr reflektieren sie auch über Maßnahmen zum Wiederaufbau von Staatlichkeit und mahnen zu Vorsicht gegenüber dem Drängen alter und neuer Machthaber auf die Etablierung einer abermals militarisierten Sicherheitsarchitektur, die arbeitslose männliche Jugendliche vor dem Kriegsausbruch in paramilitärische Gruppen einband. Comfort Ero gibt in ihrem Aufsatz »Security Sector Reform. Re-Imagining its Transformative Potential« (2011) zu bedenken, dass nationale und lokale Eliten, die sich beispielsweise in Liberia und Sierra Leone auf den Traditionserhalt und die Wiederherstellung der Ordnung berufen, die Forderungen dortiger sozial marginalisierter Frauen und junger Männer nach Würde, Menschenund Landrechten ignorieren würden. 
In ihrem Beitrag »Security Sector Reform and Women's Peace Activism Nexus in Liberia « hält Ecoma Alaga (2011) den Planern der UN-Friedensmission und der Entwaffnungs- und Demobilisierungsprogramme in Liberia einen Spiegel vor und prangert deren Ignoranz gegenüber den lokalen Realitäten an. Sie deckt die geringen Einflussmöglichkeiten der rangniedrigen Gender-Beraterin in der UN-Friedensmission auf und dämpft die hohen Erwartungen, die an die indischen Polizistinnen gestellt wurden. Anstatt sie als Vorbilder für den Umbau des liberianischen Polizeiapparats zu verklären, illustriert die Friedensforscherin die Fortsetzung des Sexismus und die Willkür innerhalb der Polizei und Justiz.

Auch die Beiträge in dem von den deutschen Politologinnen Bettina Engels und Corinna Gayer Ende 2011 herausgegebenen Sammelband »Geschlechterverhältnisse, Frieden und Konflikt« (2011 a) setzen sich mit geschlechtsspezifischen Machtverhältnissen und gesellschaftlichen Differenzen nach Kriegen auseinander. In ihrem Aufsatz »Kriegsveteranen in der Friedensarbeit - militarisierte Männlichkeit als Friedenspotenzial?« ergründet Miriam Schroer-Hippel (2011) die Motive und maskulinen Selbstbilder kroatischer Kriegsveteranen, die sich in zivilgesellschaftlichen Friedensinitiativen engagieren. Unter Bezug auf eigene empirische Forschungen und lokale Studien zeigt sie auf, wie die Veteranen militärische Männlichkeitsbilder neu interpretieren, um als patriotische Bürger auftreten zu können. In diesen Friedensinitiativen wird über Täter- und Opferzuschreibungen, Kriegsursachen, Nationalismus und friedliche Zukunftsperspektiven verhandelt. Dabei geraten Geschlechterstereotypen teilweise ins Wanken und vor allem Männlichkeitsvorstellungen müssen neu definiert werden. Die Psychologin erkennt die friedensstiftenden Potenziale und die Erweiterung von Maskulinitätszuschreibungen durch die Initiativen an, bleibt aber gleichzeitig kritisch gegenüber nationalistischen Interpretationen der Kriegsursachen.

Die anderen Autorinnen und Autoren des Sammelbandes »Geschlechterverhältnisse, Frieden und Konflikt « stellen ebenfalls Ergebnisse ihrer empirischen und theoriegeleiteten Studien vor. Den konzeptionellen Rahmen dazu bieten die Herausgeberinnen, indem sie in ihrem erkenntnisreichen Einleitungsaufsatz die Entwicklung der hiesigen feministischen Friedens- und Konfliktforschung seit den 1980er Jahren erklären. In diesem Kontext erläutern sie ihr Verständnis von Gender als Analysekategorie von Macht- und Herrschaftsverhältnissen. Sie verlangen kritische Selbstreflexionen über eigene Grundannahmen, insbesondere mit Blick auf eurozentrische und reduktionistische Erklärungsmuster. Darüber hinaus entfalten Engels und Gayer in ihrem Aufsatz »Feministische Perspektiven in der Friedensund Konfliktforschung « (2011 b) das aktuelle Forschungsspektrum zwischen Em- 
pirie und Theorie sowie zwischen Wissenschaft und Praxis, in das alle Beiträge ihres Sammelbands eingeordnet werden können.

Deren Bandbreite reicht von Geschlechterbildern deutscher Bundeswehrsoldaten (Dittmer 2011), der Berichterstattung über den Afghanistankrieg (Nachtigall/Bewernitz 2011), Geschlecht und Terror am Beispiel von Selbstmordattentaten über militante malayisch-muslimische Untergrundkämpfer in Südthailand (Streicher 2011) bis zu konfliktfördernden bzw. -bewältigenden Interventionen von Frauenorganisationen in Kenia (Daniel 2011) und Gender in der Übergangsjustiz (BuckleyZistel/Zolkos 2011). Die AutorenInnen reflektieren sowohl über gesellschaftliche oder politische Strukturprobleme im Kontext von Kriegen und gewaltsamen Konflikten, als auch über das praktische Vorgehen, die Ergebnisse und möglichen Folgen der eigenen Untersuchungen.

\section{Erkenntnisse lokaler Wissenschaftlerinnen und Positionierungen}

Solche grundlegenden Fragen betreffen Wissenschaftlerinnen in Post-Konfliktländern noch stärker, zumal sie direkt mit den Kriegsfolgen und den Herausforderungen der Friedensprozesse konfrontiert sind. Umso beachtlicher ist ihre analytische Tiefenschärfe, die Differenzierungen und kritische politische Stellungnahmen beinhaltet. Sie nutzen Gender-Ansätze als Analyseinstrument, um Konflikte, Friedensprozesse und sicherheitspolitische Probleme zu untersuchen. Ihre historisch, politisch und ökonomisch kenntnisreichen Studien zeugen vom Erkenntnisgewinn durch die systematische Integration von Gender-Perspektiven. Einblicke in solche Studien gewährt der von Funmi Olonisakin und Awino Okech herausgegebene Sammelband »Women and Security Governance in Africa«.

Feministische Friedensforscherinnen in unterschiedlichen Ländern nehmen auf derartige Studien Bezug, um zu dokumentieren, dass die Forschung nicht nur in Europa oder in den USA stattfindet, sondern WissenschaftlerInnen aus Nachkriegsgesellschaften selbst zu den Forschenden zählen. So rezipieren beispielsweise auch die in der Aufsatzsammlung »Geschlechterverhältnisse, Frieden und Konflikt « vereinten Autorinnen im Zuge ihrer selbstkritischen Standortbestimmungen Publikationen von Forscherinnen aus Nachkriegsländern.

Wie kritisch afrikanische Autorinnen ihre eigenen Gesellschaften und politischen Systeme nach Kriegen durchleuchten, dokumentiert der Sammelband »Women and Security Governance in Africa « besonders eindrücklich. In bestechend scharfen Problemanalysen entlarven sie den Wiederaufbau der Patronagenetze alter Eliten auf nationaler und lokaler Ebene. Die Binnensicht, die das dichte Geflecht zwischen geschlechtsspezifischer Gewalt und politischen bzw. wirtschaftlichen Gewalt-, 
Ausbeutungs- und Unterdrückungsformen auf lokaler und nationaler Ebene seziert, straft hoffnungsvolle Einschätzungen ab. Dies betrifft beispielsweise Liberia, das einige europäische und US-amerikanische Konfliktforscher gern als Paradebeispiel für erfolgreiche Nachkriegsentwicklungen einstufen. Eine genaue Gender-Analyse lokaler Wissenschaftlerinnen wie Ecoma Alaga deckt jedoch die Bedeutung des Sicherheitsapparats für den Machterhalt der politischen Eliten auf. Denn schon vor dem Bürgerkrieg in den 1990er Jahren nutzten diese geschlechtsspezifische Gewalt als inhärenten Bestandteil der Herrschaftsabsicherung - ein Erbe, das keineswegs aufgearbeitet ist (Alaga 2011).

Allen hier vorgestellten Büchern ist gemeinsam, dass sie die Misere von Frauen und die Gewaltprobleme in Nachkriegsgesellschaften nicht isoliert betrachten, sondern die komplexen Interdependenzen von Gewaltstrukturen auf der Mikro- und Makroebene erkennen. Insbesondere Gender-Analysen, die kriegsgeprägte Männlichkeit und deren vielschichtigen Folgewirkungen beachten, tragen maßgeblich zum umfassenden Verständnis von gewaltsamen Konflikten und den Schwierigkeiten in Friedensprozessen bei. Deshalb wäre es wünschenswert, wenn sich die hiesige etablierte Friedens- und Konfliktforschung mit diesen Studien intensiver auseinandersetzen würde. 


\section{Literatur}

Abdela, Lesley 2010: Nepal and the Implementation of UNSCR 1325, in: Olonisakin et al. 2010, 66-86.

Alaga, Ecoma 2011: Security Sector Reform and Women's Peace Activism Nexus in Liberia, in: Olonisakin/Okech 2011, 68-88.

Anderlini, Sanam 2010: Translating Global Agreements into National and Local Commitments, in: Kuehnast et al. 2010, 19-36.

Brück, Thomas/Vothknecht, Marc 2010: Impact of Violent Conflict on Women's Economic Opportunities, in: Kuehnast et al. 2010, 85-114.

Buckley-Zistel, Susanne/Zolkos, Magdalena 2011: Das Gender von Gerechtigkeit nach Krieg, Gewalt und Unterdrückung. Geschlechterdimensionen der Transitional Justice, in: Engels/Gayer 2011 a, 135-149.

Daniel, Antje 2011: Frauenorganisationen in der Postkonfliktsituation in Kenia im Spannungsfeld zwischen neuen und tradierten Handlungsräumen, in: Engels/ Gayer 2011 a, 113-132.

Dittmer, Cordula 2011: »Sie wissen ja, wie Frauen so sein können...«. Feministisch forschen in der Bundeswehr?, in: Engels/Gayer 2011 a, 81-94.

Engels, Bettina/Gayer, Corinna (Hrsg.) 2011 a: Geschlechterverhältnisse, Frieden und Konflikt. Feministische Denkanstöße für die Friedens- und Konfliktforschung, Baden-Baden.

Engels, Bettina/Gayer, Corinna 2011 b: Feministische Perspektiven in der Friedensund Konfliktforschung, in: dies. 2011 a, 9-26.

Ero, Comfort 2011: Security Sector Reform. Re-Imagining its Transformative Potential, in: Olonisakin/Okech 2011, 31-48.

Ikpe, Eka 2011: ECOWAS, Women and Security, in: Olonisakin/Okech 2011, 124-144.

Kuehnast, Kathleen/de Jonge Oudraat, Chantal/Hernes, Helga (Hrsg.) 2010: Women and War - Power and Protection in the 21st Century, Washington, DC.

Murithi, Tim 2011: The African Union and Security Governance, in: Olonisakin/ Okech 2011, 145-160.

Nachtigall, Andrea/Bewernitz, Torsten 2011: Von >FrauenundKindern` zu >Embedded Feminism ‘. Frauen(rechte) als Legitimation für militärische Interventionen in den Medien - Variationen einer Legitimationsfigur zwischen Kosovo-, Afghanistan- und Irakkrieg, in: Engels/Gayer 2011 a, 27-46.

Nakaya, Sumie 2010: Women and Gender Issues in Peacebuilding. Lessons Learned from Timor-Leste, in: Olonisakin et al. 2010, 155-169. 
Olonisakin, Funmi/Barnes, Karen/Ikpe, Eka (Hrsg.) 2010: Women, Peace and Security. Translating Policy into Practice, London.

Olonisakin, Funmi/Okech, Awino (Hrsg.) 2011: Women and Security Governance in Africa, Cape Town.

Scanlon Helen/Nhalevilo Benilde 2011: Many Thruths Were Not Revealed. The Case of Mozambique, in: Olonisakin/Okech 2011, 108-122.

Schroer-Hippel, Miriam 2011: Kriegsveteranen in der Friedensarbeit-militarisierte Männlichkeit als Friedenspotenzial?, in: Engels/Gayer 2011 a, 95-112.

Sidi Bah, Mohamed 2011: Opportunities, Challenges and Lessons Learned for Women, in: Olonisakin/Okech 2011, 89-107.

Skjelsbaek, Inger 2010: Sexual Violence in the Post-Yugoslav War, in: Kuehnast et al. 2010, 19-36.

Streicher, Ruth 2011: Die Macht des Feldes. Selbstreflexivität als methodologische Strategie feministischer Forschung in Konfliktgebieten, in: Engels/Gayer 2011 a, 65-79.

Wood, Elisabeth 2010: Rape Is Not Inevitable During War, in: Kuehnast et al. 2010, 37-64.

\section{Die Autorin}

Dr. Rita Schäfer ist freiberufliche Wissenschaftlerin.

E-Mail: info@frauen-und-kriege-afrika.de 